\title{
Morbid obesity is not a contraindication to lower limb arthroplasty
}

\author{
Martin SHARrock, Ashwani Nugur, Saqif Hossain
}

From the Department of Trauma and Orthopaedic Surgery, The Royal Oldham Hospital, Northern Care Alliance, Manchester, UK

There are concerns that increased BMI is associated with a greater length of stay (LOS) and perioperative complications following total knee (TKR) and total hip replacements (THR).

We analysed data from a six-month period to see if there was a correlation between BMI and LOS. We performed a subgroup analysis for patients with morbid obesity (BMI >40) looking at perioperative complications.

285 TKRs and 195 THRs were analysed. For TKRs, the average length of stay was 2.7 days. The average BMI was 32.4. There was no significant correlation between BMI and $\operatorname{LOS}(r=-0.0447, p=0.2267)$. The morbidly obese category $(n=33)$ had the shortest LOS (2.5 days) compared to other BMI categories. 30day readmission rate was $6 \%$. 90-day re-admission rate was $12 \%$. Six patients had minor wound issues requiring no intervention or antibiotics only. The was one prosthetic joint infection, one stitch abscess, one DVT and one patellar tendon injury.

For THRs, the average LOS was 2.9 days. The average BMI was 29.9. There was no significant correlation between BMI and $\operatorname{LOS}(r=0.007, p=0.4613)$. The morbid obese category $(n=9)$ had the shortest LOS (1.9 days) compared to other BMI categories. No patients were readmitted within 90 days or had documented complications.

We have shown that for TKRs and THRs, increased BMI is not associated with increased LOS. The morbidly obese were found to have the shortest LOS. Re-admission rates and complications were commendable for patients with morbid obesity. BMI $>40$ is not a contraindication to TKR or THR.

No benefits or funds were received in support of this study. None of the authors have a conflict of interest.
Keywords : arthroplasty ; hip ; knee ; replacement ; morbid obesity ; body mass index ; BMI.

\section{INTRODUCTION}

Obesity is a significant risk factor for the development of osteoarthritis (1). We are seeing a rise in the proportion of lower limb arthroplasty procedures performed on patients with a high body mass index (BMI). The National Joint Registry's (NJR) 2019 annual report states that the average person undergoing a total knee replacement (TKR) has a BMI of 30.7 (obese), and the average BMI of a patient undergoing a total hip replacement (THR) is 28.7 (overweight) (2).

Obesity is a multi-systemic disease, often linked with diabetes, cardiovascular and pulmonary comorbidities. As such there are concerns that an increased

Martin SHARROCK, MBChB, BSc, MRCS

Ashwani NUGUR, MBBS, DNB

- Saqif HOSSAIN, FRCS (Glas), FRCS (Eng), FRCS (Tr \& Orth)

Department of Trauma and Orthopaedic Surgery, The Royal Oldham Hospital, Northern Care Alliance, Manchester, United Kingdom

Correspondence : Martin Sharrock, Department of Trauma and Orthopaedic Surgery, The Royal Oldham Hospital, Northern Care Alliance, Manchester, United Kingdom.

Email : martin.sharrock@doctors.org.uk

- 2021, Acta Orthopædica Belgica. 
BMI is associated with perioperative complications including venous thromboembolism (VTE), postoperative deep and superficial infections, airway difficulty, dislocations, revisions, and mortality $(3,4)$. The increased risk of complications, together with the functional challenges involved with rehabilitating obese patients, raises concerns that increased BMI is associated with a longer length of stay (LOS) following lower limb arthroplasty $(5,6)$.

Data from the NJR states that for patients undergoing total hip and knee replacements at our trust, the BMI is higher than the national average (2). Our objective was to test the hypothesis that increased BMI was associated with increased length of stay and perioperative complications. The primary aim of this paper is to assess the effect BMI has on length of stay following primary hip and knee replacements. The secondary aim of this paper was to perform a subgroup analysis for patients with a BMI greater than 40 to analyse whether this category of patient is at risk of perioperative complications.

\section{METHODS}

Data was collected retrospectively on all patients who had a primary total hip or knee replacement within our trust over a six-month period (February 2019 to August 2019). Our trust serves a population of 820,000 in the North-East sector of Greater Manchester. We collected data from 12 lower limb arthroplasty surgeons. Data was available via our online patient records.

We performed a qualitative subgroup analysis on patients with a BMI of greater than 40 . We looked at patient age, operation time, haemoglobin drop, 30-day and 90-day readmission rate, range of motion at final clinical review and postoperative complications. Patients were followed up for at least one year.

Statistical analysis was performed using GraphPad Prism 8.4.2. Normality was assessed by the Shapiro-Wilk test. Correlations between BMI and LOS were computed using Spearman's rank correlation. Comparisons between mean LOS for each BMI category was calculated using one-way ANOVA (Kruskal-Wallis) with multiple comparisons. Statistical significance was set at $\mathrm{p}<0.05$.

\section{RESULTS}

Over the six-month period 495 patients underwent total hip or knee arthroplasty at our unit. Six patients had no recorded BMI and were excluded. One patient had bilateral total knee replacements and was excluded. Eight patients had a LOS of over 10 days which were considered extreme outliers and henceforth excluded from the main analysis. This left 195 THRs and 285 TKRs for analysis.

The average length of stay for patients undergoing TKR was 2.7 days. The average BMI was 32.4. There was no significant correlation between BMI and $\operatorname{LOS}(\mathrm{r}=-0.0447, \mathrm{p}=0.2267)$ as seen on figure 1. A non-significant reduction in LOS can be seen as BMI increases. When comparing BMI categories there was no significant difference $(p=0.7116)$ as seen on figure 2 and table 2. Patients with a BMI greater than 40 had the shortest LOS.

The average length of stay for patients undergoing THR was 2.9 days. The average BMI was 29.9. There was no significant correlation between BMI and $\operatorname{LOS}(\mathrm{r}=0.007, \mathrm{p}=0.4613)$ as seen on figure 1. A non-significant increase in LOS can be seen as BMI increases. When comparing BMI categories there was no significant difference $(\mathrm{p}=0.4613)$ as seen on figure 2 and table 1 . Patients with a BMI greater than 40 had the shortest LOS.

33 patients had a BMI greater than 40 . Two of these had a LOS over 10 days and were excluded from the prior analysis but are included here as part of the descriptive analysis. The average age was 65 years. The average operative time was 78 minutes (data only available for 24 patients). The average haemoglobin drop was $2.4 \mathrm{~g} / \mathrm{dL}$ and no patients required blood transfusion.

Two patients $(6 \%)$ were readmitted within 30 days. Four patients $(12 \%)$ were readmitted within 90 days. One patient was admitted on post-operative day five with cellulitis and treated with antibiotics for five days. Another patient was admitted six weeks post-operatively with a stitch abscess that required incision and drainage. Two patients had suspected deep vein thrombosis; one was excluded 

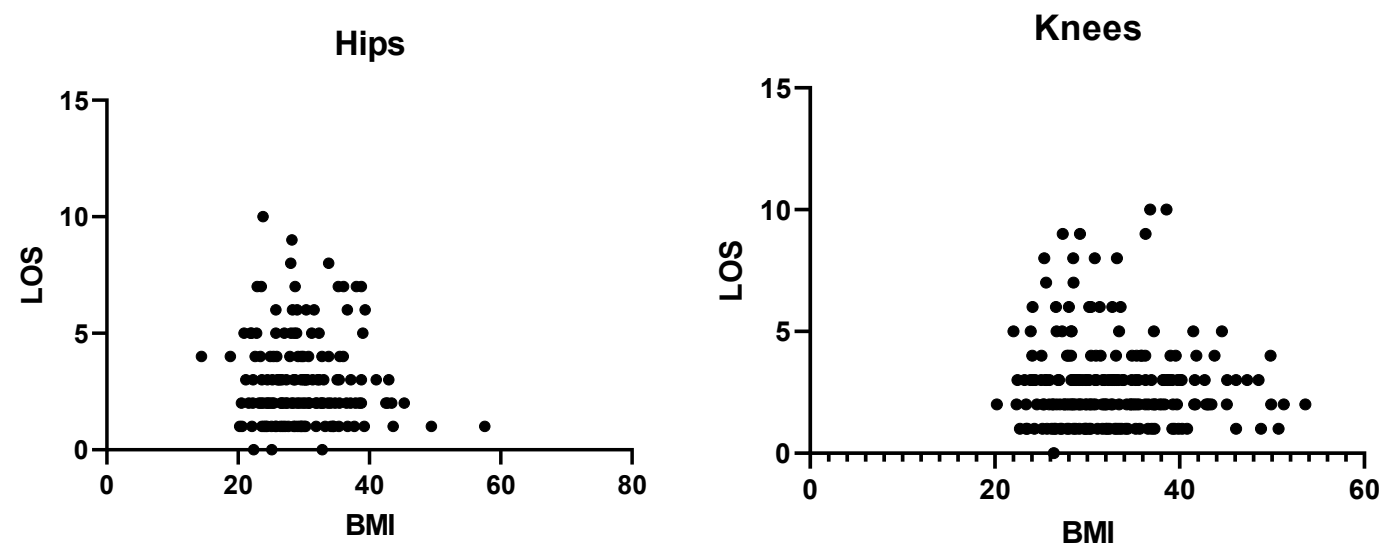

Figure 1. - Scatter graphs showing correlation between BMI and LOS for total hip and knee replacements.
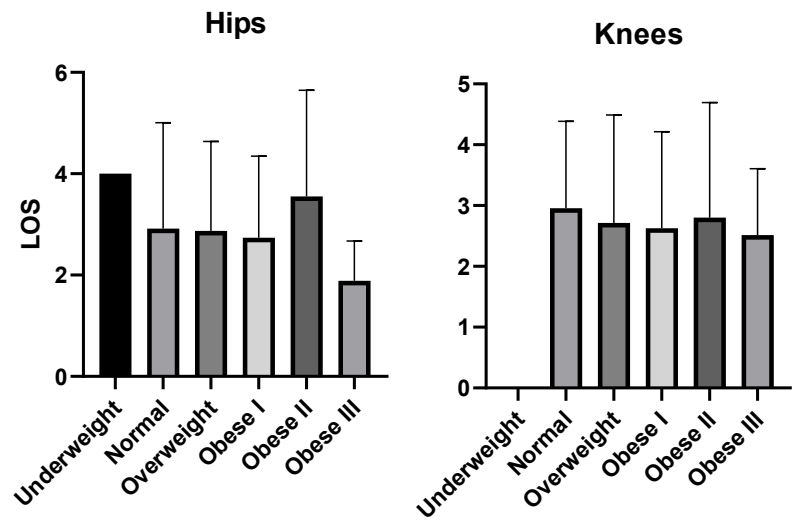

Figure 2. - Bar charts showing mean (and standard deviation) LOS for each BMI category. Underweight, BMI $<18.5$. Normal, BMI 18.5-24.9. Overweight, BMI 25-29.9. Obese I, BMI 3034.9. Obese II, BMI 35-39.9. Obese III, BMI $>40$.

Table 1. - Mean LOS for each BMI category for total hip replacements

\begin{tabular}{|l|l|c|c|}
\hline BMI & Category & $\begin{array}{c}\text { Number of } \\
\text { patients }\end{array}$ & Mean LOS \\
\hline$<18.5$ & Underweight & 1 & 4.0 \\
\hline $18.5-24.9$ & Normal & 36 & 2.9 \\
\hline $25-29.9$ & Overweight & 78 & 2.9 \\
\hline $30-34.9$ & Obese I & 42 & 2.7 \\
\hline $35-39.9$ & Obese II & 29 & 3.6 \\
\hline$>40$ & Obese III & 9 & 1.9 \\
\hline
\end{tabular}

on Doppler, the other had an equivocal Doppler and was discharged on apixaban for three months. Median follow up was 17 months (range 12-19)

Range of motion was measured at the final clinical follow up. $30(91 \%)$ patients could flex
Table 2. - Mean LOS for each BMI category for total knee replacements

\begin{tabular}{|l|l|c|c|}
\hline BMI & Category & $\begin{array}{c}\text { Number of } \\
\text { patients }\end{array}$ & Mean LOS \\
\hline$<18.5$ & Underweight & 0 & $\mathrm{n} / \mathrm{a}$ \\
\hline $18.5-24.9$ & Normal & 22 & 3.0 \\
\hline $25-29.9$ & Overweight & 98 & 2.7 \\
\hline $30-34.9$ & Obese I & 78 & 2.6 \\
\hline $35-39.9$ & Obese II & 56 & 2.8 \\
\hline$>40$ & Obese III & 31 & 2.5 \\
\hline
\end{tabular}

Table 3. - Complication rates for patients with a BMI greater than 40 undergoing total knee replacement

\begin{tabular}{|l|c|}
\hline Complication & Number \\
\hline $\begin{array}{l}\text { Minor wound issue (required antibiotics or no } \\
\text { intervention) }\end{array}$ & 6 \\
\hline Urine retention & 2 \\
\hline Prosthetic joint infection (diagnosed at 5 months) & 1 \\
\hline Stitch abscess requiring incision and drainage & 1 \\
\hline Deep vein thrombosis & 1 \\
\hline Unsatisfactory range of motion & 1 \\
\hline Intraoperative patellar tendon injury & 1 \\
\hline
\end{tabular}

beyond 90 degrees. Only four patients couldn't reach full extension. One patient had a range of motion of 0-70 and underwent manipulation under anaesthesia and arthrolysis. Complications can be seen on table 3.

9 patients had a BMI greater than 40 . The average age was 63 years. The average operative time was 118 minutes. The average haemoglobin drop was 2.2 $\mathrm{g} / \mathrm{dL}$ and no patients required blood transfusion. No patients were readmitted within 90 days. No patients 
had any documented complications. Median follow up was 16 months (range 12-18).

\section{DISCUSSION}

The results from our primary analysis show that increased BMI is not associated with increased LOS following primary total hip or knee replacements. For TKRs there was a non-significant weak negative correlation between BMI and LOS. For THRs there was a non-significant weak positive correlation between BMI and LOS. For both TKRs and THRs, patients with a BMI of greater than 40 had the shortest length of stay compared to other BMI categories (2.5 days for knees, 1.9 days for hips).

Previous research looking at the effect of BMI on LOS following TKR has yielded conflicting results. Prohaska et al. (2017) analysed 716 primary TKRs performed at a single institute in the United States of America and concluded that increased BMI was correlated with increased LOS and that BMI over 40 was associated with discharge to institution (6). Keeney et al. (2019) supported this by showing that, in patients with a BMI greater than 40, losing 9 kilograms preoperatively lead to reduced LOS and reduced discharge to institution following TKR (7). On the contrary, Husted et al. (2016) and Al-Azzani et al. (2017) showed that BMI is not associated with increased LOS $(8,9)$. Chen et al. (2016) demonstrated that although patients undergoing TKR with a BMI of greater than 40 has a longer LOS and 30-day readmission rate, they had a greater improvement in functional outcomes at two year follow up (10).

With regards to THR, again the literature is conflicting. Hung et al. (2019) showed that increased age, operative time and presence of cardiovascular comorbidities were associated with greater 30-day complications, but failed to demonstrate a link between BMI and LOS or 30-day complications (11). Richard et al. (2018) and Iqbal et al. (2016) both showed that BMI does not affect LOS following THR $(12,13)$. On the other hand, Bradley et al. (2014) and Kremers et al. (2014) found a correlation between increased BMI and both LOS and cost $(5,14)$. Husted et al. (2016) found that BMI greater than 35 was associated with LOS over four days (8).
There are concerns that operating on the morbidly obese may lead to inferior outcomes. Barrett et al. (2018) performed a systematic review of THRs comparing BMI greater than 35 with BMI less than 35 , finding increased revision rates with a higher BMI $\quad(7.99 \%$ versus $2.75 \%$, respectively) (15). Nevertheless they also demonstrated a comparable improvement in functional outcomes scores between morbidly obese and non-obese patients (15).Contrary to this however, Chee et al. (2010) identified that functional outcomes improved more if the patient was non-obese (16). Chee et al. (2010) also noted increased complications and reduced five-year survivorship in the morbidly obese (16). With respect to TKRs, Amin et al. (2006) showed that BMI greater than 40 was associated with lower Knee Society Scores, increased complications and inferior survivorship (17). Halawi et al. (2019) showed that patient-reported outcomes were only inferior in the six months immediately postoperative for patients with a BMI of greater than 40 ; beyond six months there was no difference (18). They also found no difference in patient-reported outcomes following THR when comparing BMI greater than 40 with BMI less than 40 (18).

30-day readmission rates following TKR (for all BMI categories combined) have been quoted at $3.3-4.7 \%$, and 90 -day readmissions quoted at 8.2$9.7 \%(19,20)$. Top reasons for readmission include post-operative infection, cellulitis, periprosthetic fracture, wound disruption, cardiac events, VTE and pneumonia (19). It has been noted in the literature that morbid obesity has been associated with higher readmission rates $(21,22)$. In our series of patients with a BMI of greater than 40 , we identified a 30-day readmission rate of $6 \%$ and a 90 -day re-admission rate of $12 \%$. We do note that one of our readmissions was for a suspected DVT that was ruled out and could therefore be seen as an inappropriate admission. Although readmissions for the morbidly obese in our series were slightly higher than other BMI categories quoted in the literature, we feel that the relatively low readmission rate in this 'high-risk' category should be commended.

30-day readmission rates following THR (for all BMI categories combined) have been quoted at 3.4\%-5.6\%, and 90-day readmissions quoted 
at $7.2 \%-7.7 \%(19,20)$. Common reasons for readmission are similar to TKR, but also include dislocation (19). In our series of morbidly obese patients we had no readmissions within 90 days, although we do note our sample size of nine is limited.

Lübbeke et al. (2016) analysed 9,061 TKRs and THRs and noted prosthetic joint infection rates to be $1.1 \%$ if BMI was less than $35,2.1 \%$ if BMI was $35-40$, and $3.7 \%$ if BMI was greater than 40 (23). In the current study we identified one prosthetic joint infection out of 42 lower limb arthroplasties $(2.4 \%)$ performed in the patients with a BMI greater than 40 . In our series we found that prosthetic joint infection rates in the morbidly obese are roughly double that of a 'typical' arthroplasty patient, which is lower than quoted in the literature.

The most frequency complication we found with patients with a BMI of greater than 40 was issues with the wound, all in TKRs. We identified six (14.3\%) minor wound issues (two oozing wounds, two issues with skin clips, one superficial wound infection and one cellulitis). One patient required incision and drainage of a stitch abscess at two months post-TKR. Wound issues have been noted to be more frequent with increasing BMI elsewhere in the literature $(24,25)$. Given the increased risk of deep and superficial infections in patients with a BMI of greater than 40, it is essential that when consenting these patients we make it clear they are fully informed of their higher complication risk.

The literature is conflicting regarding the effect of BMI on development of postoperative VTE. Anecdotally obesity is associated with greater risk of VTE, however there are several large studies that show obese patients are at no greater risk than nonobese patients (25-27). In the general population, it has been suggested that $1.3 \%$ of patients undergoing a THR and $2.8 \%$ of patients undergoing a TKR will develop VTE (28). It is important to point out that these figures vary throughout the literature, with Sadeghi et al. (2012) identifying that $21 \%$ of patients undergoing TKR in their series developed VTE (26).The rate of VTE was low (2.4\%) in our analysis of patients with a BMI of greater than 40 , suggesting that increased BMI doesn't affect VTE risk.
The results from our subgroup analysis of patients with a BMI greater than 40 show that surgical complications are similar to those quoted in the literature for all patients undergoing lower limb arthroplasty.

A limitation of our study is that we lack long term follow up. Our median follow up for morbidly obese patients is 16 months and therefore we cannot provide long term data on revision rates or implant survivorship ; for which there are concerns about in this BMI category (15-17).

Another limitation of our data is the relatively small number of patients with a BMI of greater than 40 (33 knees, 9 hips) however we do note that our average BMI is higher than the average quoted in the NJR's 2019 annual report (2). For TKRs, the NJR average BMI is 30.7, however our trust average BMI is 32.4 (2). For THRs, the NJR average BMI is 28.7, whereas ours is 29.9 (2). It could be hypothesised that the short LOS and reasonable complication rates for patients with a BMI of greater than 40 in our series is due to our increased exposure to patients with a high BMI. If surgeons are regularly performing arthroplasty in patients with a higher than average BMI then this could lead to improved outcomes in this cohort of patients. One of the key findings of the Getting It Right First Time report was that a high number of surgeons were performing fewer than ten revision cases per year, and a potential solution for this is to set up revision centres for surgeons to subspecialise solely in revision arthroplasty (29). Given that the incidence of obesity related arthritis is increasing, would it be unreasonable to suggest in future years we see surgeons subspecialising in arthroplasty in the morbidly obese?

\section{CONCLUSION}

We have shown that for primary TKRs and THRs, increased BMI is not associated with increased LOS. The morbidly obese category was found to have the shortest LOS, although this difference wasn't statistically significant. Re-admission rates and complications for the morbidly obese were comparable to the literature for non-obese 
patients. We feel that BMI greater than 40 is not a contraindication to TKR or THR.

\section{REFERENCES}

1. King LK, March L, Anandacoomarasamy A. Obesity \& osteoarthritis. Indian J Med Res. 2013 Aug ; 138(2) : 185193.

2. National Joint Registry. $16^{\text {th }}$ Annual Report [Internet]. 2019 [cited 15/9/2020]. Available from: https://reports. njrcentre.org.uk/

3. Sabharwal S, Root MZ. Impact of obesity on orthopaedics. J Bone Joint Surg A. 2012 Jun ; 94(11) : 1045-1052.

4. Parratte S, Pesenti S, Argenson JN. Obesity in orthopedics and trauma surgery. Orthop Traumatol Surg Res. 2014 Feb ; 100(1 Suppl) : S91-S97.

5. Bradley BM, Griffiths SN, Stewart KJ, Higgins GA, Hockings M, Isaac DL. The effect of obesity and increasing age on operative time and length of stay in primary hip and knee arthroplasty. J Arthroplasty. 2014 Oct ; 29(10) : 19061910.

6. Prohaska MG, Keeney BJ, Beg HA, et al. Preoperative body mass index and physical function are associated with length of stay and facility discharge after total knee arthroplasty. Knee. 2017 Jun ; 24(3) : 634-640.

7. Keeney BJ, Austin DC, Jevsevar DS. Preoperative Weight Loss for Morbidly Obese Patients Undergoing Total Knee Arthroplasty : Determining the Necessary Amount. J Bone Joint Surg Am. 2019 Aug ; 101(16) : 1440-1450.

8. Husted H, Jørgensen CC, Gromov K, Kehlet H. Does BMI influence hospital stay and morbidity after fast-track hip and knee arthroplasty? Acta Orthop. 2016 Oct ; 87(5) : 466-472.

9. Al-Azzani WAK, Iqbal HJ, Al-Soudaine Y, et al. Impact of BMI and ASA grade on length of stay following primary total knee replacement. Orthop Proceed Bone Joint J. 2017 Apr ; 99B(Suppl) : 8.

10. Chen JY, Lo NN, Chong HC, et al. The influence of body mass index on functional outcome and quality of life after total knee arthroplasty. Bone Joint J. 2016 Jun ; 98-B(6) : 780-785.

11. Hung CY, Chang CH, Lin YC, Lee SH, Chen SY, Hsieh PH. Predictors for Unfavorable Early Outcomes in Elective Total Hip Arthroplasty: Does Extreme Body Mass Index Matter? BioMed Research International. 2019 Oct ; 2019 : 4370382.

12. Richard B, Reilly A, Lyden E, Garvin K. Is obesity a risk factor for extended length of stay and readmission after total hip arthroplasty? Ann Joint. 2018 Sept ; $3: 73$.

13. Iqbal HJ, Al-Azzani WAK, Al-Soudaine Y, Suhaimi MI, John A. The impact of BMI and ASA grade on length of stay in patients undergoing primary total hip replacement. Orthop Proceed Bone Joint J. 2016 Jun ; 98B(Suppl) : 13.

14. Kremers HM, Visscher SL, Kremers WK, Naessens JM, Lewallen DG. Obesity increases length of stay and direct medical costs in total hip arthroplasty. Clin Orthop Relat Res. 2014 Apr ; 472(4) : 1232-1239.

15. Barrett M, Prasad A, Boyce L, et al. Total hip arthroplasty outcomes in morbidly obese patients: A systematic review. EFORT Open Rev. 2018 Sept ; 3(9) : 507-512.

16. Chee YH, Teoh KH, Sabnis BM, Ballantyne JA, Brenkel IJ. Total hip replacement in morbidly obese patients with osteoarthritis : results of a prospectively matched study. $J$ Bone Joint Surg Br. 2010 Aug ; 92(8) : 1066-1071.

17. Amin AK, Clayton RA, Patton JT, Gaston M, Cook RE, Brenkel IJ. Total knee replacement in morbidly obese patients. Results of a prospective, matched study. J Bone Joint Surg Br. 2006 Oct ; 88(10) : 1321-1326.

18. Halawi MJ, Gronbeck C, Savoy L, Cote MP. Effect of morbid obesity on patient-reported outcomes in total joint arthroplasty : a minimum of 1-year follow-up. Arthroplast Today. 2019 Dec ; 5(4) : 493-496.

19. Saucedo JM, Marecek GS, Wanke TR, Lee J, Stulberg SD, Puri L. Understanding readmission after primary total hip and knee arthroplasty : who's at risk? J Arthroplasty. 2014 Feb ; 29(2) : 256-260.

20. Ramkumar PN, Chu CT, Harris JD, et al. Causes and Rates of Unplanned Readmissions After Elective Primary Total Joint Arthroplasty : A Systematic Review and MetaAnalysis. Am J Orthop (Belle Mead NJ). 2015 Sep ; 44(9) : 397-405.

21. Garvin K, Lyden E, Reilly A, Richard B. Is obesity associated with hospital length of stay or readmissions? Orthop Proceed Bone Joint J. 2018 Jan ; 100B (Suppl) : 1.

22. Lehtonen EJ, Hess MC, McGwin G Jr, Shah A, GodoySantos AL, Naranje S. Risk factors for early hospital readmission following total knee arthroplasty. Acta Ortop Bras. 2018 ; 26(5) : 309-313.

23. Lübbeke A, Zingg M, Vu D, et al. Body mass and weight thresholds for increased prosthetic joint infection rates after primary total joint arthroplasty. Acta Orthop. 2016 Apr ; 87(2) : 132-138.

24. Wallace G, Judge A, Prieto-Alhambra D, de Vries F, Arden NK, Cooper C. The effect of body mass index on the risk of post-operative complications during the 6 months following total hip replacement or total knee replacement surgery. Osteoarthritis Cartilage. 2014 Jul ; 22(7) : 918927.

25. Friedman RJ, Hess S, Berkowitz SD, Homering M. Complication rates after hip or knee arthroplasty in morbidly obese patients. Clin Orthop Relat Res. 2013 Oct ; 471(10) : 3358-3366.

26. Sadeghi B, Romano PS, Maynard G, et al. Mechanical and suboptimal pharmacologic prophylaxis and delayed mobilization but not morbid obesity are associated with venous thromboembolism after total knee arthroplasty: A case-control study. J Hosp Med. 2012 Nov ; 7(9) : 665-671.

27. Tay K, Bin Abd Razak HR, Tan AH. Obesity and Venous Thromboembolism in Total Knee Arthroplasty Patients in an Asian Population. J Arthroplasty. 2016 Dec ; 31(12) : 2880-2883. 
28. Samama CM, Ravaud $P$, Parent F, Barre J, Mertl P, Mismetti P. Epidemiology of venous thromboembolism after lower limb arthroplasty : the FOTO study. $J$ Thromb Haemost. 2007 Dec ; 5(12) : 2360-2367.
29. Briggs T. Getting It Right First Time. A national review of adult elective orthopaedic services in England [Internet]. 2015 [cited 2020]. Available from: https:// gettingitrightfirsttime.co.uk/girft-reports/ 